\section{穴 Heighten Science \\ P U B L I C I T I O N S Corporation ISSN \\ 2639-6629}

Review Article

\title{
Guidelines that are just for guidance
}

\author{
Swethika Sundaravel1*, Jonathan Nieves ${ }^{2}$ and Seth Baum ${ }^{3}$ \\ 'Department of Internal Medicine, University of Miami Miller School of Medicine/Palm Beach \\ Regional Campus, USA \\ ${ }^{2}$ Department of Cardiovascular Medicine, University of Miami Miller School of Medicine/Palm \\ Beach Regional Campus, USA \\ ${ }^{3}$ Department of Integrated Medicine, Florida Atlantic University, Charles E. Schmidt College of \\ Medicine, USA
}

\begin{abstract}
*Address for Correspondence: Swethika Sundaravel, Department of Internal Medicine, University of Miami Miller School of Medicine/ Palm Beach Regional Campus, USA, Email: swethikaharini@gmail.com

Submitted: 16 March 2018

Approved: 06 April 2018

Published: 09 April 2018

Copyright: () 2018 Sundaravel S, et al. This is an open access article distributed under the Creative Commons Attribution License, which permits unrestricted use, distribution, and reproduction in any medium, provided the original work is properly cited
\end{abstract}

Check for updates

\section{Review}

Hypertension (HTN) is a widely prevalent disease across the globe. Recent reports from National Health and Nutrition Examination Surveys (NHANES) indicate that the prevalence of HTN is $29 \%$ in adults more than 18 years in the US [1]. This is about 72 million adults. Worldwide, about 1.3 billion people are affected by HTN [2]. This number is projected to increase several-fold in the coming years. Given the huge burden of this disease to the healthcare system and the many deleterious effects that can result from uncontrolled HTN, we need strong guidelines to manage the same. The recently published 2017 ACC/AHA guidelines [3] on hypertension management are very meticulous and include a comprehensive stepwise approach in treating hypertension. Here we present a summary of the major changes and a concise review of the new guidelines.

The last report on HTN guidelines was released in 2003 by JNC 7 [4] which was followed by a focused update in 2014 by JNC 8 [5]. These guidelines did not include any systematic review, nor did they mention the levels of evidence. Also, due to the lack of strong randomized controlled trials for lower blood pressure (BP) targets at that time, the JNC 8 was perhaps lenient with the goals of treatment for hypertension. Since then we have had several new trials proving the benefits of more stringent blood pressure control. In synch with the growing body of clinical research data and stronger evidence for a lower BP target, the 2017 ACC/AHA guidelines on hypertension management was released right in time and it addresses all the important questions about hypertension management, also allowing some room for shared decision making and a personalized medicine approach for individual patient needs.

The authors focused on 4 critical questions which form the basis to treat HTN. They included 1. Evidence about self-monitoring of BP in terms of preventing adverse outcomes and to achieve better control on BP; 2. Optimal target for BP therapy; 3. Benefits and harms of various anti-HTN medications and 4. Monotherapy vs dual therapy while initiating management of HTN. There is more emphasis on ambulatory BP monitoring, home based BP monitoring and tele-medicine.

The definition of HTN has been revised in the new guideline, reducing the threshold for HTN from $140 / 90 \mathrm{mmHg}$ to anything over $130 / 80 \mathrm{mmHg}$. The classification of HTN is also changed now, stage 1 being $>130 / 80 \mathrm{mmHg}$ to $<140 / 90 \mathrm{mmHg}$ and stage 2 being $>140 / 90 \mathrm{mmHg}$. According to the new guideline, the estimated prevalence of hypertension is about 103.3 million adults in the United States which is almost $45.6 \%$ of total population [6]. Of note, the authors also define 'High Blood Pressure' as $\geq 120 / 80 \mathrm{mmHg}$. For the first time, we have clear guidelines on how to measure the BP accurately in the office setting. 
This includes very specific details such as patient positioning, adequate rest before measurement, cuff size, repeat readings with average values, BP recording in both arms, BP recording techniques, and even the admonition for physicians not to be the ones taking readings, to avoid potentially falsely elevated readings. Issues about "white coat HTN" and "masked HTN" for which there have not been any clear guidelines so far, have been clarified. Previously, we had inconsistencies with management of white coat hypertension; some physicians treated it as typical hypertension while others assessed the burden of hypertension throughout the day by performing ambulatory BP monitoring. The new guidelines recommend ambulatory BP monitoring for all patients with inconsistent BP readings. This advice is based on a wide variety of supporting data ranging from systematic reviews to meta-analyses [7-9] emphasizing the prevalence of white-coat hypertension. Screening for secondary HTN has also been adjusted, from $<40$ years to $<30$ years, likely leading to enhanced vigilance among clinicians.

In terms of management, the recommendation now is to target a lower BP goal for all patients regardless of their age or comorbidities, the new target being $130 / 80 \mathrm{mmHg}$. This is quite different from the JNC 8's advice, which supported a higher goal of $<150 / 90 \mathrm{mmHg}$ for patients over 60 years. While this has spurred a substantial debate over the past several years, with strong data from the SPRINT trial [10] supporting lower BP target, the new target should be implemented very cautiously. Although there is overwhelming evidence from RCTs like HYVET study [11] and the subgroup analysis from SPRINT [12] for patients older than 75 years supporting intensive BP control, as a primary care physician, how comfortable are we to target this goal in our old and frail patients who are at risk for falls (some of whom may be on anticoagulation therapies)? For a patient who is accustomed to the previous target of $<150 / 90 \mathrm{mmHg}$, the new lower target may be intolerable and may make them prone to side-effects from medications including light-headedness and falls (although these were not significantly increased in the SPRINT trial).

We should understand that the SPRINT trial was not the real-world population as it excluded patients with several conditions, the most important ones being diabetes mellitus, stroke, $\mathrm{HFrEF}$ with $\mathrm{EF}<35 \%$, patients with uncontrolled hypertension with systolic $>180 \mathrm{mmHg}$, proteinuria $>1 \mathrm{~g} /$ day, recent heart failure hospitalization and GFR $<20 \mathrm{~mL} / \mathrm{min} / 1.73 \mathrm{~m}^{2}$. A large number of our office patients experience one or more of these exclusion criteria. Also, to be noted is the number medications used in the intensive treatment arm. Only $10 \%$ of patients in the intensive arm were on single agent. About $30 \%$ were on 2 agents, $31 \%$ were on 3 agents and a striking $25 \%$ of patients were on 4 or more agents to achieve the intensive BP target. Can this be achieved in the real world? Will the need to increase the number of medications concomitantly augment medication non-compliance? Serious adverse events like hypotension requiring assistance and acute renal injury were reported in a significant number of patients, however, the authors concluded saying that the benefits of fewer cardiovascular events and mortality rates outweigh the risk of adverse events. It should also be noted that the number needed to treat to prevent 1 primary outcome (defined as myocardial infarction, other acute coronary syndromes, stroke, heart failure, or death from cardiovascular causes) was 61 and to prevent death from cardiovascular causes was as high as 172 . Attaining this target of $<130 / 80 \mathrm{mmHg}$ can be easier in younger adults but it could be almost impossible in older adults. Can our frail and elderly patients tolerate the new target of $<130 / 80 \mathrm{mmHg}$ ? This is where shared decision making and 'thinking outside the guidelines' will be most useful. There is no guidance on treatment of isolated systolic hypertension which is very commonly seen in older individuals. Targeting SBP of $<130 \mathrm{mmHg}$ may drop their DBP so low that it could theoretically be detrimental to coronary perfusion, causing more harm than good [13].

While the basis of this new target $<130 / 80 \mathrm{mmHg}$ stems from the SPRINT trial (the authors of which are also part of this guideline committee), several other studies 
were also analysed. This included a large meta-analysis [14] of approximately 45,000 patients from 19 other trials, which also support intensive BP control. The ACCORD trial [15], which included diabetic patients as well, did not show any clear benefit from aggressive BP control in the primary analysis, although it was a smaller trial with only half the number of patients as SPRINT, and it was also underpowered. However, there was a trend favouring intensive BP control in a sub-group analysis.

New in this guideline is the introduction of ASCVD risk into the anti-HTN therapy equation. Pharmacotherapy for HTN should be initiated based on the ASCVD risk score, which in turn considers the patient's age, sex, and history of diabetes, BP (if treated or untreated), smoking status, total cholesterol and HDL cholesterol. The ASCVD risk score has been used to predict the 10-year risk of cardiovascular events and stroke for a long time. Most of our treatment guidelines including that for diabetes and hyperlipidaemia [16] strongly recommend treatment based on the ASCVD risk score. Now, the management of HTN is also cantered around the ASCVD risk score. It is recommended to start pharmacotherapy in all patients with stage 1 HTN with co-existent CVD or ASCVD risk $>10 \%$. In the absence of CVD, non-pharmacological therapy should be used as an initial approach. For stage 2 HTN pharmacotherapy is indicated even in the absence of CVD. For patients with ASCVD risk $<10 \%$, a softer style is recommended with initiation of pharmacotherapy only at BP $>140 / 80 \mathrm{mmHg}$. This integration of ASCVD risk with hypertension management is a welcome change as hypertension is one of the major contributors to ASCVD risk. The use of a risk calculator reinforces the common goal in managing these associated conditions, which is, to prevent cardiovascular mortality.

Non-pharmacological measures including lifestyle modifications, weight loss, dietary modifications such as DASH diet (Dietary Approaches to Stop Hypertension), reduction in alcohol consumption and regular physical activity are still class 1 recommendations for the management of hypertension. This too reinforces the importance of therapeutic lifestyle changes (TLC) across all risk or disease state. TLC is the foundation of optimal health. Physical activity such as aerobic exercises, dynamic resistance training and isometric resistance training have been shown to be extremely beneficial for hypertension management as elucidated by several meta-analyses $[17,18]$. Exercise training is also one of the cornerstones in the management of other co-morbid conditions like obesity, diabetes mellitus and hyperlipidaemia as all these conditions often tend to co-exist as the metabolic syndrome [19]. Among dietary modifications, the new guidelines emphasize on salt restriction and enhanced potassium intake both of which are proven to be very useful in hypertensive patients [20].

Among pharmacological agents, the major change is the elimination of beta-blockers (BB) as first line agents. According to the new guidelines angiotensin converting enzyme inhibitors/aldosterone receptor blockers (ACEI/ARB), calcium channel blocker (CCB) and thiazides are the first line agents. BB are the first line agents only when there are other indications. For patients with baseline BP 20/10 mmHg above goal, it is recommended to start with 2 agents. For African-American patients, CCB or thiazide diuretics are still the preferred agents over ACEI or ARB unless there is coexisting heart failure or chronic kidney disease. Follow up is recommended in 1 month for all patients. This quick follow-up time-frame also reinforces the gravity of HTN and the need to manage it aggressively.

Ischemic heart disease is a very common result of uncontrolled HTN. The new guidelines recommend a goal BP $<130 / 80 \mathrm{mmHg}$ and guideline directed BB (includes carvedilol, metoprolol tartrate, metoprolol succinate, nadolol, bisoprolol propranolol, and timolol) as the class 1 agents for HTN in patients with stable ischemic heart disease, then calcium channel blockers and then finally diuretics. Atenolol is now eschewed as an anti-hypertensive agent as it is less effective than placebo in reducing cardiovascular events [21]. 
HTN can lead to several other diseases, one of the most important conditions being heart failure. There was no target BP goal for heart failure in the previous guidelines. Now, it is recommended to target a goal of $<130 / 80 \mathrm{mmHg}$ for management of HTN in heart failure with reduced or preserved ejection fraction. The use of non-di hydro pyridine calcium channel blockers in HFrEF is an absolute contraindication (class 3). Other agents can be used based on the other comorbidities, however, BB and ACEI or ARB should be used routinely as they have mortality benefit in heart failure with reduced EF. For HFpEF, there are no documented benefits in terms of mortality for the various agents. It should be noted that a majority of HFpEF patients initially present with hypertensive crisis and acute pulmonary edema [22]. In the setting of HFpEF and volume overload, it is recommended to use diuretics to control HTN (class 1c). Otherwise, ACEI or BB are the agents of choice for treating HTN in HFpEF. Although the TOPCAT study [23] showed a trend towards mortality benefit with spironolactone in patients with HFpEF in North and South America, it has not yet been adopted by guidelines. More recently, an interesting retrospective observational study was published in JAMA cardiology that showed low systolic blood pressure $(<120 \mathrm{mmHg}$ at discharge) was linked to worse outcomes in HFpEF with HR of 2.07 (95\% CI, 1.452.95; P <.001) for 30-day all-cause mortality [24]. Is there a sweet spot to target? If so, what is it, and is it pragmatically achievable in clinical practice? Another downstream effect of long standing HTN is chronic kidney disease (CKD). The new guideline also recommends a goal of $<130 / 80 \mathrm{mmHg}$ for treating HTN in CKD, which is the same as JNC 8. In the absence of proteinuria, any agent can be used. In the presence of proteinuria, ACEI/ARBs should be first line agents (class 2a). Up to a 30\% increase in creatinine can occur in such patients after starting ACEI/ARB.

HTN is also common after renal transplantation, owing to the use of immune suppressants like calcineurin inhibitors and corticosteroids. The goal here should be $<130 / 80 \mathrm{mmHg}$. Calcium channel blockers are the agents of choice (class 2a). This recommendation is based on a Cochrane analysis [25] which found reduced graft loss and preserved GFR in patients treated with CCBs. Additionally, it showed that the use of ACEI is harmful in renal transplant patients. Although this is not a guideline by itself, the writers suggest that caution should be exercised while using ACEI for HTN management in post-transplant patients. In the presence of other comorbidities such as heart failure or proteinuria, ACEI can be used.

Cerebrovascular disease is the number one cause of death in patients with HTN. Hypertensive patients are prone to both ischemic and haemorrhagic strokes. In the setting of acute intra-cranial haemorrhage ( $<6$ hours), it is recommended not to intervene if the BP is between $150-220 \mathrm{mmHg}$ systolic, as this can potentially be harmful. The use of intra-venous agents to reduce BP is recommended for systolic BP $>220 \mathrm{mmHg}$.

For ischemic stroke, the guidelines haven't significantly changed. The management of HTN is based on whether the patient is a candidate for thrombolytic therapy or not, as tPA can be used only if the BP is $<185 / 110 \mathrm{mmHg}$. After thrombolytic therapy, BP should be maintained $<180 / 105 \mathrm{mmHg}$. For patients who do not undergo thrombolysis or endovascular intervention, permissive HTN of up to $220 / 110 \mathrm{mmHg}$ for 24 hours should be allowed. Trying to correct this could be potentially harmful. Beyond $220 / 110 \mathrm{mmHg}$, it is reasonable to lower the BP by $15 \%$ in the first 24 hours after stroke. For secondary stroke prevention, the general goal BP is $<130 / 80 \mathrm{mmHg}$ after the acute phase. Thiazide diuretics or AEI/ARBs or a combination of both can be used.

For Peripheral Arterial Disease (PAD), it is recommended to control the BP to a goal of $<130 / 80 \mathrm{mmHg}$ just like any other patient. PAD is considered a CAD equivalent and demands aggressive risk factor modification. There are no specific recommendations in terms of agents of choice. Contrary to the popular belief that beta blockers should be avoided in PAD, it was shown in the INVEST [26] and ALLHAT [27] trials that there is no difference in outcomes between these and other agents when used for HTN management. 
Type 2 Diabetes Mellitus is an important comorbid condition found in many patients with HTN. The combination of HTN and diabetes places patients at extreme risk of injury to the systemic vasculature and kidneys. Therefore, it is necessary to aggressively control both conditions. The BP goal in diabetic patients should be $<130 / 80 \mathrm{mmHg}$. This recommendation is based on 2 meta-analyses which studied the optimal BP targets in diabetic patients [28,29]. Although the ACCORD trial did not demonstrate any benefit in intensive BP control, the study was underpowered. A subset analysis from the study, did reveal significantly reduced primary outcomes in the intensive BP control and standard glycaemic control group. Although SPRINT did not include any diabetic patients, it did show that, in pre-diabetics, the benefit of intensive control of BP was far greater than lenient control. Any agent can be used in the absence of proteinuria while, in the presence of proteinuria, ACEI/ARB is recommended for superior renal protection. For metabolic syndrome, although there are still no clear guidelines, vasodilating betablockers like labetalol, nebivolol and carvedilol had favourable effects on metabolic profile compared to standard agents. To date, there are no RCTs evaluating this population.

The JNC 8 made no recommendations about treating hypertension in atrial fibrillation. The new guideline recommends using ARBs for treating hypertension in such patients as they have also been found to prevent recurrent atrial fibrillation. This recommendation is based on the trials which studied RAS blockade with ARB in comparison to BB and CCB $[30,31]$. There are no studies evaluating the effect of ACEI.

In terms of HTN management in valvular heart diseases, we have clear guidelines for aortic stenosis and aortic insufficiency. It is recommended to start at low doses and gradually increase the dose of anti-HTN medications for patients with aortic stenosis. Diuretics should be sparingly used in patients with a small left ventricular chamber and aortic stenosis as such patients are pre-load dependent, (although there are no studies supporting this recommendation). For aortic insufficiency, it is recommended to avoid agents that lower the heart rate, such as BB and CCB, as they prolong the diastolic phase and may thereby augment the regurgitate volume.

For acute aortic syndromes such as aortic dissection, BB are still the first line agents to control the BP. Hypertension management in pregnancy remains the same. The preferred medications are nifedipine, methyl-dopa, hydralazine and labetalol (class 1 recommendation). ACEI and ARBs are contra-indicated (class 3).

Peri-operative hypertension management remains the same, except for some clear guidance on use of peri-operative $\mathrm{BB}$, which was a grey area previously. The recent guideline recommends continuing $\mathrm{BB}$ peri-operatively in patients who were previously taking BB. It also recommends against initiating BB therapy peri-operatively in BB naïve patients (class 3-harm). This advice is based on several observational studies and the POISE trial, which was a large RCT [32].

The new guideline emphasizes ambulatory BP monitoring and tele health, including health personnel contacting patients about their BP readings and advising medication titration in between office visits. Though this recap of the salient aspects of the recent hypertension guideline is important, we should understand that guidelines are simply a 'guide' to physicians who are managing various disease states. Guidelines should never dictate management in a vacuum. Guidelines should be applied to patients at an individual level and decisions should be made in discussion with the patient considering his/her preferences, particularly in vulnerable populations such as the elderly. Such discussions and considerations might result in deviation from the guidelines. This is okay. Evidence based medicine should be practiced in a patient centric manner, which will make us better physicians, treating healthier patients. 


\section{References}

1. Cheryl D. Fryar, Yechiam Ostchega, Craig M. Hales, Guangyu Zhang, Deanna Kruszon-Moran Hypertension Prevalence and Control among Adults: United States, 2015-2016. NCHS Data Brief No. 289. 2017. Ref.: https://goo.gl/rLMioj

2. Michael J. Bloch. Worldwide prevalence of hypertension exceeds 1.3 billion. Journal of the American Society of Hypertension. 2016; 10: 753-754. Ref.: https://goo.gl/xPou25

3. Whelton PK, Robert Carey M, Wilbert Aronow S, Donald Casey E, Karen Collins J, et al. ACC/AHA/ AAPA/ABC/ACPM/AGS/APhA/ASH/ASPC/NMA/PCNA Guideline for the Prevention, Detection, Evaluation, and Management of High Blood Pressure in Adults. 2017 High Blood Pressure Clinical Practice Guideline. 2017. Ref.: https://goo.gl/cza7TM

4. Aram V. Chobanian, George L. Bakris and the National High Blood Pressure Education Program Coordinating Committee. Seventh Report of the Joint National Committee on Prevention, Detection, Evaluation, and Treatment of High Blood Pressure. Hypertension. 2003; 42: 1206-1252. Ref.: https://goo.gl/Qzx9RS

5. Paul A. James, Suzanne Oparil, Barry L. Carter. 2014 Evidence-Based Guideline for the Management of High Blood Pressure in Adults Report from the Panel Members Appointed to the Eighth Joint National Committee (JNC 8). JAMA. 2014; 311: 507-520. Ref.: https://goo.gl/PSAeNN

6. Muntner P, Carey RM, Gidding S, Jones DW, Taler SJ, et al. Potential US population impact of the 2017 ACC/ AHA high blood pressure guideline. Circulation. 2018; 137: 109-118. Ref.: https://goo.gl/P3unf8

7. Fagard RH, Cornelissen VA. Incidence of cardiovascular events in white-coat, masked and sustained hypertension versus true normotension: a meta-analysis. J Hypertens. 2007; 25: 2193-2198. Ref.: https://goo.gl/5bqkGM

8. Piper MA, Evans CV, Burda BU, Margolis KL, O'Connor E, et al. Diagnostic and predictive accuracy of blood pressure screening methods with consideration of rescreening intervals: a systematic review for the U.S. Preventive Services Task Force. Ann Intern Med. 2015; 162: 192-204. Ref.: https://goo.gl/crDByQ

9. Bayo J, Cos FX, Roca C, Dalfó A, Martín-Baranera MM, et al. Home blood pressure self-monitoring: diagnostic performance in white-coat hypertension. Blood Press Monit. 2006; 11: 47-52. Ref.: https://goo.gl/pJ1BPe

10. SPRINT Research Group, Wright JT Jr, Williamson JD, Whelton PK, Snyder JK, et al. A randomized trial of intensive versus standard blood-pressure control. N Engl J Med. 2015; 373: 2103-2116. Ref.: https://goo.gl/gpXUo9

11. Warwick J, Falaschetti E, Rockwood K, Mitnitski A, Thijs L, et al. No evidence that frailty modifies the positive impact of antihypertensive treatment in very elderly people: an investigation of the impact of frailty upon treatment effect in the HYpertension in the Very Elderly Trial (HYVET) study, a doubleblind, placebo-controlled study of antihypertensives in people with hypertension aged 80 and over. BMC Med. 2015; 13: 78. Ref.: https://goo.gl/nEZntP

12. Williamson JD, Supiano MA, Applegate WB, Berlowitz DR, Campbell RC, et al. Intensive vs standard blood pressure control and cardiovascular disease outcomes in adults aged $\geq 75$ years: a randomized clinical trial. JAMA. 2016; 315: 2673-2682. Ref.: https://goo.gl/VwuA22

13. Steven Tringali, Charles William Oberer, Jian Huang. Low Diastolic Blood Pressure as a Risk for AllCause Mortality in VA Patients. Int J Hypertension. 2013; 2013. Ref.: https://goo.gl/JFNa3Y

14. Xie X, Atkins E, Lv J, Bennett A, Neal B, et al. Effects of intensive blood pressure lowering on cardiovascular and renal outcomes: updated systematic review and meta-analysis. Lancet. 2016; 387: 435-443. Ref.: https://goo.gl/Fm9wCG

15. ACCORD Study Group, Cushman WC, Evans GW, Byington RP, Goff DC Jr, et al. Effects of intensive blood-pressure control in type 2 diabetes mellitus. N Engl J Med. 2010; 362: 1575-1585. Ref.: https://goo.gl/Yo2Ecr

16. Stone NJ, Robinson J, Lichtenstein AH, Bairey Merz CN, Blum CB, et al. 2013 ACC/AHA Guideline on the Treatment of Blood Cholesterol to Reduce Atherosclerotic Cardiovascular Risk in Adults: A Report of the American College of Cardiology/American Heart Association Task Force on Practice Guidelines. J Am Coll Cardiol. 2014; 63: 2889-2934. Ref.: https://goo.gl/MGXmXw

17. Cornelissen VA, Smart NA. Exercise training for blood pressure: a systematic review and metaanalysis. J Am Heart Assoc. 2013; 2: e004473. Ref.: https://goo.gl/wJ5pLF

18. Carlson DJ, Dieberg G, Hess NC, Millar PJ, Smart NA. Isometric exercise training for blood pressure management: a systematic review and meta-analysis. Mayo Clin Proc. 2014; 89: 327-334. Ref.: https://goo.gl/XMBQFF 
19. Garcia-Hermoso A, Saavedra JM, Escalante Y. Effects of exercise on resting blood pressure in obese children: a meta-analysis of randomized controlled trials. Obes Rev. 2013; 14: 919-928. Ref.: https://goo.gl/dJXQNk

20. Whelton PK, He J. Health effects of sodium and potassium in humans. Curr Opin Lipidol. 2014; 25: 75-79. Ref.: https://goo.gl/avJ8X7

21. Carlberg B, Samuelsson O, Lindholm LH. Atenolol in hypertension: is it a wise choice? Lancet. 2004; 364: 1684-1689. Ref.: https://goo.gl/3dbN6D

22. Kalkidan Bishu and Margaret M. Redfield. Acute Heart Failure with Preserved Ejection Fraction: Unique Patient Characteristics and Targets for Therapy. Curr Heart Fail Rep. 2013; 10. Ref.: https://goo.gl/dkGXA5

23. Pfeffer MA, Claggett B, Assmann SF, Boineau R, Anand IS, et al. Regional variation in patients and outcomes in the Treatment of Preserved Cardiac Function Heart Failure with an Aldosterone Antagonist (TOPCAT) trial. Circulation. 2015; 131: 34-42. Ref.: https://goo.gl/96n4Wr

24. Fraction Apostolos Tsimploulis, Phillip H. Lam, Cherinne Arundel, et al. Systolic Blood Pressure and Outcomes in Patients with Heart Failure with Preserved Ejection. JAMA Cardiol.

25. Cross NB, Webster AC, Masson P, O'Connell PJ, Craig JC. Antihypertensive treatment for kidney transplant recipients. Cochrane Database Syst Rev. 2009; CD003598. Ref.: https://goo.gl/nKsZ9e

26. Bavry AA, Anderson RD, Gong Y, Denardo SJ, Cooper-Dehoff RM, et al. Outcomes among hypertensive patients with concomitant peripheral and coronary artery disease: findings from the INternational VErapamil-SR/Trandolapril STudy. Hypertension 2010; 55: 48-53. Ref.: https://goo.gl/Xytj4P

27. Antihypertensive and Lipid-Lowering Treatment to Prevent Heart Attack Trial Collaborative Research Group. Diuretic versus alpha-blocker as first-step antihypertensive therapy: final results from the Antihypertensive and LipidLowering Treatment to Prevent Heart Attack Trial (ALLHAT). Hypertension. 2003; 42: 239-246. Ref.: https://goo.gl/TnDUyv

28. Emdin CA, Rahimi K, Neal B, Emdin CA, Rahimi K, Neal B, et al. Blood pressure lowering in type 2 diabetes: a systematic review and meta-analysis. JAMA. 2015; 313: 603-615. Ref.: https://goo.gl/ruUWgp

29. Arguedas JA, Leiva V, Wright JM. Blood pressure targets for hypertension in people with diabetes mellitus. Cochrane Database Syst Rev. 2013; 10: CD008277. Ref.: https://goo.gl/XQspJW

30. Wachtell K, Lehto M, Gerdts E, Olsen MH, Hornestam B, et al. Angiotensin II receptor blockade reduces new-onset atrial fibrillation and subsequent stroke compared to atenolol: the Losartan Intervention for End Point Reduction in Hypertension (LIFE) study. J Am Coll Cardiol. 2005; 45: 712719. Ref.: https://goo.gl/k527KL

31. Julius S, Kjeldsen SE, Weber M, Brunner HR, Ekman S, et al. Outcomes in hypertensive patients at high cardiovascular risk treated with regimens based on valsartan or amlodipine: the VALUE randomised trial. Lancet. 2004; 363: 2022- 2031. Ref.: https://goo.gl/SLTGSf

32. Devereaux PJ, Yang H, Yusuf S, Guyatt G, Leslie K, et al. Effects of extended-release metoprolol succinate in patients undergoing noncardiac surgery (POISE trial): a randomised controlled trial. POISE Study Group. Lancet. 2008; 371: 1839-1847. Ref.: https://goo.gl/2a1ZrD 